35

\section{THE INCIDENCE OF CORONARY ARTERY ABNORMALITY IN INCOM- PLETE KAWASAKI DISEASE}

Tomoyoshi Sonobe ${ }^{1}$, Seijiro Aso ${ }^{1}$, Yoshio Imada ${ }^{1}$, Keiji Tsuchiya ${ }^{1}$, Yosikazu Nakamura ${ }^{2}$, Hiroshi Yanagawa ${ }^{3}$ Department of Pediatrics, Japanese Red Cross Medical Center, Tokyo, Japan ${ }^{1}$, Department of Public Health, Jichi Medical School, Tochigi, Japan², Saitama Prefectural University, Saitama, Japan ${ }^{3}$

Purpose: To clarify the incidence of coronary artery abnormality (CAA) among incomplete (atypical) Kawasaki disease.Method: We defined incomplete cases as follows. The incomplete Kawasaki disease of narrow definition (nd-IKD) consists of cases with 4 items of principal symptoms without CAA, and cases with less than 4 items of principal symptoms regardless of CAA. The broad definition of incomplete Kawasaki disease (bd-IKD) consists of cases with less than 5 items of principal symptoms regardless of CAA. About 48,000 cases of Japanese nationwide Kawasak disease survey were analyzed using our criteria. The proportion, age distribution and the incidence of CAA of incomplete cases were compared with that of typical cases with 5 to 6 items of principal symptoms. Result: The proportion of typical cases, bd-IKD and nd-IKD were $85.0 \%, 15.0 \%$ and $11.5 \%$, respectively. The frequency of incomplete cases was slightly higher among the age groups of less than 1 year and over 5 year. The incidence of acute phase CAA among typical cases, bd-IKD and nd-IKD were $19 \%, 20 \%$ and $5 \%$, respectively. The incidence of CAA of 30th illness day among typical cases, bd-IKD and nd-IKD were $12 \%, 11 \%$ and $2 \%$, respectively. Discussion: Although the proportion of CAA in nd-IKD was smaller than that of typical cases, the proportion of CAA in bd-IKD was almost same as that of typical cases. Therefore the same treatment is required for the cases with less than 5 items of principal symptoms. The revision of diagnostic guideline may be necessary when the above mentioned evidence is confirmed by precise secondary survey.

\section{6}

DOES THE ADMINISTERED SALICYLATE DOSE PREDICT IVIG TREATMENT FAILURE IN KAWASAKI DISEASE ?

Sheri Fisher, Nancy Innocentini, Stanford T. Shulman Pediatric Infectious Diseases, Children's Memorial Hospital, Northwestern University Medical School, Chicago, IL, USA

A sub-group of KD patients fails initial IVIG treatment, develops persistent or recurrent fever and inflammatory signs, and require repeat IVIG or other therapy. Factors contributing to IVIG treatment failure are incompletely understood. We hypothesized that administering lower aspirin doses contributes to initial IVIG treatment failure. Methods: From our database of $394 \mathrm{KD}$ patients treated from 1992-2001, we identified those who received more than one $2 \mathrm{gm} / \mathrm{kg}$ dose of IVIG. Each was matched with two KD controls by gender, age and illness day at initial IVIG treatment. Data regarding clinical and echocardiographic features, aspirin dosing, and laboratory findings were were assessed and compared for retreated patients and matched controls. Results: 26 patients $(6.5 \%)$ had been treated with more than one $2 \mathrm{gm} / \mathrm{kg}$ dose of IVIG, including 20 who were retreated once and six retreated twice. Fifty matched controls were identified. There was no significant difference between patients and matched controls with respect to age, gender, day of illness of initial treatment, and pre-treatment WBC, absolute band count, hemoglobin, albumin, and ESR. Retreated patients had significantly higher pre-preatment absolute neutrophil counts $(\mathrm{P}=0.008)$ and total bilirubin levels $(\mathrm{P}=0.009)$. No differences in KD presenting clinical features between retreated patients and matched controls, except that peripheral desquamation was noted more often in retreated patients $(p=0.043)$. Although marked differences in aspirin dosing were not observed, a significantly greater proportion of retreated patients than matched controls were initially treated with $<80 \mathrm{mg} / \mathrm{kg}$ ASA $(35 \%$ vs. $12 \%$, respectively $[\mathrm{P}=0.022]$ on the first treatment day, $36 \%$ vs. $12 \%$ on the second treatment day $[\mathrm{p}=0.020]$, and $32 \%$ vs. $14 \%$ on the third treatment day $[\mathrm{P}=0.086])$. Retreated patients had abnormal echocardiograms more foten than mathed controls $(\mathrm{p}=0.027)$, mainly reflecting baseline abnormalities. We conclude that lower administered initial doses of ASA may contribulte to IVIG treatment failure and thus to consequent need for retreatment and possibly poorer echocardiographic outcomes.

\section{7}

RANDOMIZED TRIAL OF PULSE STEROIDS IN THE INITIAL TREATMENT OF KAWASAKI DISEASE (KD)

Robert P Sundel ${ }^{1}$, Annette L Baker ${ }^{2}$, David R Fulton ${ }^{2}$, Jane W Newburger ${ }^{2}$ Division of Immunology (Rheumatology), Children's Hospital, Boston, MA, USA ${ }^{1}$, Department of Cardiology, Children's Hospital, Boston, MA, USA ${ }^{2}$

We conducted a prospective randomized trial to determine whether addition of corticosteroids to IVIG might improve outcomes. Subjects met AHA criteria for KD and were treated at Childrens Hospital, Boston, between July 1998 to July, 2000 during the fi rst 10 days of fever. Subjects were randomized to receive IVIG, $2 \mathrm{gm} / \mathrm{kg}$ over 10 hours, with or without pulse IVMP, $30 \mathrm{mg} / \mathrm{kg}$ (maximum $1.5 \mathrm{gm}$ ) over $3 \mathrm{hrs}$. All patients received aspirin, 20 to $25 \mathrm{mg} / \mathrm{kg} \mathrm{q} 6 \mathrm{~h}$ until afebrile for 48 hours, then $3-5 \mathrm{mg} / \mathrm{kg} / \mathrm{day}$. Twenty subjects received IVMP plus IVIG, 21 received IVIG alone. Groups were similar in baseline demographic, laboratory data, and coronary artery measurements. The mean duration of fever after starting IVIG therapy was $0.9 \pm 1.2$ days (mean \pm SD) in the IVMP group, $2.4 \pm 1.9$ days in the IVIG alone group $(\mathrm{P}=0.005$, unpaired $\mathrm{t}$-test). Patients treated with IVMP plus IVIG had shorter hospital stays $(2.0 \pm 0.8$ vs. $3.3 \pm 2.1$ days, $P=0.007)$, and at six weeks, lower ESR $(10.6 \pm 6.0$ vs.. $19.4 \pm 12.4, \mathrm{P}=0.02)$ and lower mean $\ln \mathrm{CRP}(-3.27 \pm 0.64$ vs $-2.38 \pm 1.16, \mathrm{P}=.01)$. No significant differences between treatment groups were noted in coronary dimensions at two or six weeks. IVMP was well tolerated; one child developed transient hypertension. In summary, treatment of acute KD with IVMP plus IVIG, compared to IVIG alone, resulted in faster resolution of fever, more rapid improvement in markers of inflammation, and shorter length of hospitalization. Adverse effects were infrequent. The effect of steroid therapy on coronary artery outcome, however, remains unproven. Further study of the optimal use of steroids in the treatment of KD should be performed.

\section{8}

CORTICOSTEROIDS ADD-ON THERAPY IN THE ACUTE PHASE OF KAWASAKI DISEASE

Sun-mi Kang ${ }^{1}$, Su-mi In ${ }^{2}$, Eun-Kyung Moon ${ }^{1}$, Hong-Ryang Kil ${ }^{1}$ Department of Pediatrics, Chungnam National University Hospital, Daejeon, Korea ${ }^{1}$, Department of Pediatrics, Daejeon Sun Hospital, Mok-Dong, Jung-gu, Daejeon, Korea

Purpose: Recently the clinical trials of steroid add-on therapy were reported with variable results in Kawasaki disease. We analyzed clinical outcome of high risk of patients with Kawasaki disease( $\geq$ 4 points of Harada score) treated by 3 commonly used different treatment regimen with or without corticosteroids. Methods: Medical records of 96 children with Kawasaki disease treated with one of the 3 regimens were reviewed retrospectively. Regimen 1 was aspirin $(100 \mathrm{mg} / \mathrm{kg} / \mathrm{day})$ plus intravenous gamma globulin $2 \mathrm{~g} / \mathrm{kg}$ single dose; regimen 2 , aspirin ( $100 \mathrm{mg} / \mathrm{kg} /$ day) plus intravenous gamma globulin $1 \mathrm{~g} / \mathrm{kg}$ single dose; regimen 3 , regimen 2 plus prednisolone $(2 \mathrm{mg} / \mathrm{kg} /$ day $)$, followed by tapering 2 weeks and pulse therapy of methyl prednisolone was performed in case of the retreatment. Also low dose aspirin was given in all 3 regimens for 8 weeks after acute phase. The cardiovascular and laboratory evaluations were performed on acute phase, immediate after acute phase, and subacute phase, 8 weeks after treatment. Results: The frequency of coronary artery lesions and laboratory findings in 3 different regimens were similar. The more rapid control of fever after treatment was noted in regimen 3 . Furthermore the frequency of retreatment was decreased in regimen 3 compared to other 2 regimens. Conclusion: Steroid add-on therapy showed some beneficial outcome compared to conventional treatment regimens. The Role of steroid in the treatment of Kawasaki disease should be reassessed in systemic manner.
39

EVALUATION OF NEW URINASTATIN THERAPY FOR THE ACUTE PHASE OF KAWASAKI DISEASE

Masao Nakano Department of Pediatrics, Gifu Prefectural Tajimi Hospital, Tajimi, Japan

Background In the therapy of Kawasaki disease (K.D.), We sometimes fail to prevent the coronary artery aneurysm formation despite high dose immunoglobulin ( $\gamma$-Gl) administration . I have already reported that polymorphonuclear leukocyte elastase(PMN-elastase) plays an important role in provoking the angitis in the acute phase of K.D. and that by giving a PMN-elastase inhibitor, urinastatin ( UTI ) in the early stage of K.D., it is able to decrease vascular injury and to prevent aneurysm formation. Aim The aim of this study is to evaluate the efficacy of new protocol of UTI therapy for the acute phase of K.D. Subjects The subjects were consecutive 44 cases with K.D. fulfilling the clinical criteria for diagnosis within 7 days of onset of illness, that had not been treated with corticosteroid, Aspirin or I $\gamma$-Gl. Methods All cases were administered 50,000U/1hr d.I.v. x 6 per day of UTI daily until the child became afebril and CRP was improved less than $1.0 \mathrm{mg} / \mathrm{dl}$. I $\gamma$-Gl was additionally administered only to the case that showed recurrence of fever and other symptoms and reincrease of CRP and WBC during UTI therapy. Results Thirty eight cases were able to be therapied by UTI alone, and six cases needed UTI and one or two times of additional $1.0 \mathrm{~g} / \mathrm{kg}$ of I $\gamma$-Gl. In only three of the six cases, echocardiographic abnormalities of coronary arteries were detected. Two cases showed mild transient dilatation and in another case, medium sized aneurysms developed. The other 41 cases (93\%) showed no coronary artery involvement. Conclusion The new UTI therapy proved to be a valuable and useful alternative for the acute phase of K.D. 